\title{
Program Adaptations to Provide Harm Reduction Services During the COVID-19 Pandemic: A Qualitative Study of Syringe Services Programs in the U.S.
}

\author{
Madeline C. Frost ${ }^{1,2}$ (1) Elsa W. Sweek ${ }^{1}$ Elizabeth J. Austin ${ }^{1} \cdot$ Maria A. Corcorran $^{3}$ - Alexa M. Juarez ${ }^{3} \cdot$ Noah D. Frank $^{3}$. \\ Stephanie M. Prohaska ${ }^{4}$ Paul A. LaKosky ${ }^{4}$ Alice K. Asher ${ }^{5}$ - Dita Broz ${ }^{6}$. Don C. Des Jarlais ${ }^{7}$ Emily C. Williams ${ }^{1,2}$. \\ Sara N. Glick ${ }^{3}$
}

Accepted: 31 May 2021 / Published online: 10 June 2021

(c) This is a U.S. government work and not under copyright protection in the U.S.; foreign copyright protection may apply 2021

\begin{abstract}
Syringe services programs (SSPs) are essential to preventing injection drug use-related infections and overdose death among people who use drugs (PWUD). The novel coronavirus (COVID-19) pandemic initially impeded SSPs' operations. To effectively support these programs, information is needed regarding SSPs' experiences adapting their services and the challenges posed by COVID-19. We conducted qualitative interviews with leadership and staff from a sample of 31 U.S. SSPs. Respondents discussed urgent concerns including reduced reach of services, suspended HIV/hepatitis C testing, high COVID-19 risk among PWUD, and negative impacts of isolation on overdose and mental health. They also noted opportunities to improve future services for PWUD, including shifting to evidence-based distribution practices and maintaining regulatory changes that increased access to opioid use disorder medications post-pandemic. Findings can inform efforts to support SSPs in restoring and expanding services, and provide insight into SSPs' role in engaging PWUD during the COVID-19 response and future emergencies.
\end{abstract}

Keywords COVID- $19 \cdot$ Coronavirus $\cdot$ Syringe services programs $\cdot$ Syringe exchange

\section{Resumen}

Los programas de servicios de jeringas (reconocido como SSP en inglés) son esenciales para prevenir las infecciones relacionadas con el consumo de drogas inyectables y la muerte por sobredosis entre las personas que consumen drogas (reconocidos como PWUD en ingles). La nueva pandemia del coronavirus (COVID-19) inicialmente impidió las operaciones de los SSP. Para apoyar eficazmente estos programas, se necesita información sobre las experiencias de los SSP que adaptan sus servicios y los desafíos que plantea COVID-19. Realizamos entrevistas cualitativas con el liderazgo y el personal de una muestra de 31 SSPs de EE.UU. Los encuestados discutieron las preocupaciones urgentes, incluyendo la reducción del alcance de los servicios, la suspensión de las pruebas de VIH/hepatitis C, el alto riesgo de COVID-19 entre la PWUD, y los impactos

Sara N. Glick

snglick@uw.edu

1 Department of Health Services, University of Washington School of Public Health, Seattle, WA, USA

2 Health Services Research \& Development Center of Innovation for Veteran-Centered and Value-Driven Care, Veterans Affairs Puget Sound Health Care System, Seattle, WA, USA

3 Department of Medicine, Division of Allergy and Infectious Diseases, University of Washington School of Medicine, Seattle, WA, USA
4 Dave Purchase Project, North American Syringe Exchange Network, Tacoma, WA, USA

5 Office of Policy, Planning and Partnerships, National Center for HIV/AIDS, Viral Hepatitis, STD and TB Prevention, Centers for Disease Control and Prevention, Atlanta, GA, USA

6 Division of HIV/AIDS Prevention, National Center for HIV/AIDS, Viral Hepatitis, STD and TB Prevention, Centers for Disease Control and Prevention, Atlanta, GA, USA

7 School of Global Public Health, New York University, New York, NY, USA 
negativos del aislamiento en las sobredosis y la salud mental. También identificaron las oportunidades de mejorar los servicios futuros para las PWUD, incluyendo el cambio a prácticas de distribución basadas en evidencias y el mantenimiento de cambios regulatorios que aumentaran el acceso a medicamentos para el trastorno por consumo de opiáceos después de la pandemia. La información que se encontró en este estudio se puede utilizar junto los esfuerzos para apoyar a los SSP en la restauración y expansión de los servicios, y proporcionar información sobre el papel de los SSP en la participación de PWUD durante la respuesta covid-19 futuras emergencias.

\section{Introduction}

The novel coronavirus (COVID-19) pandemic exacerbated multiple existing public health concerns and has significantly impacted people who use drugs (PWUD). Health leaders have called attention to the likelihood that isolation and psychosocial consequences of the pandemic may increase substance use, and that decreased access to substance use disorder (SUD) treatment and harm reduction services may increase transmission of drug use-related infections and worsen the overdose crisis [1-3]. Provisional data from the Centers for Disease Control and Prevention [4, 5], emergency department records [6, 7], and media reports from over 40 U.S. states [8] suggest that overdose deaths have risen sharply during the pandemic.

Syringe services programs (SSPs) were established to prevent HIV transmission by distributing safe injection drug use equipment [9], and research has demonstrated SSPs' effectiveness in preventing transmission of HIV and other blood-borne pathogens [10]. Their role has expanded to include delivery of multiple treatment and harm reduction services, including testing and treatment for HIV and hepatitis $\mathrm{C}$ virus (HCV) [11], distribution of naloxone and fentanyl test strips to prevent overdose death $[12,13]$, harm reduction services for noninjection drug use (e.g., safe smoking equipment) [14], and provision of medication for opioid use disorder (MOUD) [15, 16]. SSPs may also advocate to decrease substance use-related stigma and increase the wellbeing of PWUD through community education and policy change [17, 18].

Two assessments of U.S. SSP services conducted early in the COVID-19 pandemic found that many programs had closed, and those that remained open had significantly reduced their services $[19,20]$. A recently published qualitative study of representatives from 18 SSPs enrolled in a randomized controlled trial testing naloxone distribution practices found that these programs had demonstrated resilience and developed innovative strategies to continue operating during the pandemic [21]. Additional information about service adaptations from a broad sample of SSPs is needed to effectively support these programs in continuing to provide life-saving services, as well as to inform SSPs' role in the pandemic response among a population that may be at particularly high risk for contracting and experiencing hospitalization and/or death from COVID-19 [22] and in preparing for future emergencies. We conducted semi-structured qualitative interviews with leadership and staff from a sample of U.S. SSPs to understand: 1) SSP staff and leadership's understanding of and reaction to COVID19-related rules and guidelines enacted in their community, 2) strategies employed to implement safety precautions and associated barriers, 3 ) perceptions of the pandemic's impact on SSP services and other impacts on PWUD, and 4) perspectives regarding how the pandemic created opportunities to improve services for PWUD in the future.

\section{Methods}

\section{Study sample and recruitment}

The sample included four SSPs located in cities with high COVID-19 infection rates early in the pandemic that were purposively sampled for our initial rapid assessment [19], plus an additional sample of randomly selected SSPs. As it was not feasible to recruit all U.S. SSPs, stratified random sampling was used an as efficient means of selecting a geographicallydiverse sample from a large pool of SSPs whose perspectives were considered equally valuable to this analysis, not as a means of achieving generalizability. The sampling frame included all known U.S. SSPs in the North American Syringe Exchange Network (NASEN) directory as of February 2020 (i.e., prior to any COVID-19-related changes or closures; $\mathrm{N}=333$ ). A random sample of 50 SSPs was drawn from the directory, stratified by Census Bureau-designated regions. SSPs within each region were assigned a random number. The 12 SSPs with the smallest randomly-assigned numbers in the Northeast, South, and West and 13 SSPs with the smallest randomly-assigned numbers in the Midwest were selected; due to the small number of SSPs in U.S. territories, one SSP with the smallest randomly-assigned number was selected in this region. We sent an initial recruitment email to all selected SSPs. A follow-up email was sent one week later, and SSPs were called (if a telephone number was available) or sent a final email one week after the first follow-up. Any SSP staff or leadership who responded were eligible to participate. To avoid confusion with SSP participants (i.e., people who use SSP services), we refer to people who participated in interviews as "respondents." The protocol was reviewed by the University of Washington Institutional Review Board, which determined that the study was not human subjects research. 


\section{Data collection}

Semi-structured interviews were conducted remotely from 4/2/2020 to 6/25/2020 (4 in April, 15 in May, 12 in June) by four interviewers knowledgeable about SSP services (MAC, AMJ, NDF, SNG). A semi-structured interview guide (Appendix A) was developed based on expert knowledge and our initial rapid assessment [19]. Respondents provided their program's name, location and affiliation, estimated number of syringes distributed annually, and their role. Open-ended questions assessed current state/local COVID-19-related rules/guidelines, decision-making processes, changes to services, respondents' concerns, and potential opportunities to improve services. Interviews were conducted over videoconferencing software $(n=27)$ or telephone $(n=4)$. Respondents received a \$50 Visa e-gift card.

\section{Data analysis}

All interviews were recorded and transcribed verbatim. One interview was conducted in Spanish, and the transcript was translated into English. Transcripts were analyzed using a Rapid Assessment Process (RAP) [23], an intensive, iterative process that allows for rapid assessment of qualitative data in time-sensitive program evaluation contexts [24, 25]. RAP methods are more efficient than traditional qualitative methods and produce similar findings; one study comparing results from thematic analyses and RAP analyses independently conducted using the same qualitative data found that results from RAP methods had approximately $80 \%$ overlap with results from thematic analyses [26]. Therefore, RAP methods were used in order to communicate actionable results to SSPs quickly. Data from each transcript were distilled into a summary in which the respondent's answers and illustrative quotations were organized into themes based on a template. Themes were developed a priori based on the interview guide and emergent themes were added to the transcript summary template during analysis; a priori vs. emergent themes are listed in Appendix B. The template included definitions and examples and was updated by consensus of the analytic team as themes emerged throughout analysis, functioning as a standardized codebook.

Transcripts were summarized using the template by three analysts with experience in qualitative data analysis and substance use and/or HIV research (MCF, EWS, MAC). Templates were iteratively reviewed by analysts and qualitative leads with expertise in qualitative research and rapid assessment methods (EJA, ECW) who resolved discrepancies as needed. Thirtyfive percent of transcripts were independently summarized using the template by two analysts, and when clear consensus around themes had been reached remaining transcripts were each summarized by one analyst. Data from the individual transcript summaries were placed into a single matrix (displaying data within each theme across each interview); qualitative leads developed a detailed summary of themes and sub-themes which was reviewed by the entire investigative team to check conclusions against the data and identify key implications of results. A summary of findings was presented to respondents to check for accuracy. Respondents reported that the summary accurately reflected their experiences; one minor point of clarification was added to the findings.

\section{Results}

Thirty-one respondents were interviewed. SSPs varied widely in scale, ranging from 1400 to 4 million estimated syringes distributed annually. SSP and respondent characteristics are described in Table 1. Most respondents (71\%) directly provided services to SSP participants (19 program coordinators/managers, 3 nurses), and 29\% were in higherlevel leadership positions ( 8 high-level administrators and 1 state-level leader).

\section{Context and process for adapting services in response to COVID-19}

Respondents described COVID-19-related rules/guidelines and processes for making decisions and implementing changes. Some discussed how community support or lack thereof impacted SSPs' ability to adapt.

\section{The manner in which SSPs were designated essential services impacted closures}

Most respondents described their city or state as having "shut down" and/or enacted "stay-at-home orders," and were in various phases of reopening at the time of the interview. Nine SSPs had closed temporarily while awaiting essential service designation and/or determining how to adapt services; closures lasted 2-7 weeks (most lasted $\sim 2$ weeks). One SSP was still closed at the time of the interview. The manner in which SSPs were designated essential services (i.e., allowed to operate during a shut-down) by state/local government or the organization under which they operated impacted whether and for how long they closed entirely, which had immediate implications for the health and safety of participants. SSPs who received clear, rapid essential service designation generally had short closures or avoided closing, whereas some SSPs that had to seek out this designation and/or make the case for being essential had longer closures. Alternately, some SSPs decided they were essential based on their own interpretation and avoided longer closures. Health department-affiliated SSPs mostly received clear essential service designation, though some had to request this. SSPs that were nonprofit/community-based organizations had a 
Table 1 Characteristics of SSPs and respondents $(\mathrm{N}=31)^{\mathrm{a}}$

\begin{tabular}{lll}
\hline & N & $\%$ \\
\hline Geographic region & 8 & \\
Northeast & 9 & 26 \\
Midwest & 5 & 16 \\
South & 8 & 26 \\
West & 1 & 3 \\
U.S. Territories & & \\
Urbanicity/rurality & 24 & 77 \\
Urban & 7 & 23 \\
Rural & & \\
SSP type & 8 & 26 \\
Health department-operated & 23 & 74 \\
Nonprofit/community-based organization & & \\
Distribution model (pre-COVID) & 13 & 42 \\
Needs-based & 18 & 58 \\
Other & & \\
Respondent role & 19 & 61 \\
Program coordinator/manager & 8 & 26 \\
High-level administrator (e.g. executive director, & 8 & \\
$\quad$ president) & & 3 \\
Nurse & 3 & \\
State-level leader & 1 & \\
\hline
\end{tabular}

${ }^{a}$ Though some respondents discussed activities at more than one SSP site with which they were affiliated, this table describes the SSP site through which they were contacted during recruitment. Data on SSP characteristics were collected from the NASEN directory and/or respondent interviews

wider range of experiences ranging from receiving clear communication, to seeking out designation, to continuing operations without formal designation.

"We actually started advocating right away why we should not be shut down... we had to go back to our executive director and really advocate for our program. We wrote 10 pages of protocols for her to feel comfortable with us reopening. So, we had to push pretty hard for that." (Respondent 3, program coordinator/manager, urban, South)

"I never really got any sort of in black and white 'you are essential,'...We just kind of assumed we were essential, and all our staff were." (Respondent 4, program coordinator/manager, rural, Northeast)

\section{Decision-making around service adaptation often happened rapidly and internally}

Decisions regarding service adaptation happened rapidly and typically involved internal decision-making among program leadership and staff. Some programs needed to obtain external approvals before implementing changes and sometimes encountered resistance. Whether SSPs had to obtain external approval did not depend on health department affiliation - some with formal affiliations had to obtain approvals from the health department while others did not, while some nonprofit/community-based organizations had to obtain formal approvals from their director or board while others did not. Internal decision-making facilitated programs' ability to respond quickly and adjust their approach as needed, however some respondents felt the lack of a clear plan was challenging to navigate.

"...the folks like way above us, have no idea what we do, or how we do what we do...So, in terms of the decisions on how to sort of set things up and do things, it was really... a daily or weekly like, 'Okay, so we're going to try this today,'... or you know, 'This just popped up that we didn't even consider as a thing or an issue. So now we need to figure out how we're going to deal with that." ' (Respondent 5, program coordinator/manager, urban, West)

\section{Community support or lack thereof impacted the ability of some SSPs to adapt}

For some SSPs, COVID-19 adaptations were facilitated by community support or hindered by lack of support. Some programs received community donations of money, masks, and sanitizer. However, two respondents described how their community created barriers to using outdoor space to adapt services, which may have been related to stigmatization of PWUD. One described how "extremely hateful" neighbors led them to try to keep participants from waiting outside and described this as a barrier to implementing COVID-19 screening (Respondent 9, high-level administrator, urban, West), and another described how property management required them to move back inside after they had starting providing services outside (Respondent 2, nurse, urban, West). As community support was an emergent theme that was not specifically asked about by interviewers, not all respondents discussed it (Appendix B).

\section{Implementation of COVID-19 precautions and changes made to SSP services}

Precautions to prevent COVID-19 transmission included social distancing measures, use of personal protective equipment (PPE) and sanitization supplies, and, in some programs, screening participants for COVID-19 symptoms (Table 2). Some also provided COVID-19-related information or linkage to testing. 


\section{SSPs used multiple strategies to promote social distancing}

Most fixed-site SSPs (i.e., operating in one specific location) had a social distancing protocol. Many used physical cues to encourage distancing (e.g., tape, cones or signs marking six feet of distance) and had participants wait outside before coming in one or a few at a time; some conducted all distribution and returns outside or passed supplies through a window. Some respondents expressed concern that outdoor/partially outdoor models would not be sustainable in harsher weather. Contact was reduced by having outdoor sharps containers for returns, and/or having staff set down supplies and move away before participants picked them up. These measures shortened the time spent with each participant and created new demands on staff (e.g. monitoring participant entry/exit). Distancing was sometimes difficult to enforce if participants forgot or wanted to socialize.

Several programs transitioned to or increased mobile/ delivery services (i.e., providing services from a mobile location such as a van, and/or making individualized deliveries) to support distancing; some already used all-mobile/ delivery models. Distancing protocols for these models were somewhat less formal-staff no longer had participants enter vans and encouraged distancing, and a few did "no-contact" deliveries (i.e., leaving supplies in specified locations).

\section{Use of PPE and sanitization supplies was impacted by availability and perceptions of guidelines}

SSP staff routinely wore face coverings; a few programs had surgical or N95 masks, while many used cloth masks. Many SSPs had staff wear gloves, and a few wore face shields or medical gowns. Many also used hand sanitizer and sanitizing wipes to clean surfaces. Some SSPs provided face masks, gloves and/or sanitization supplies to participants, however many did not have enough supplies available to do this. Some SSPs required participants to use PPE and/or sanitizer when receiving services, others encouraged but did not require use. A few respondents discussed how inconsistency in national guidelines resulted in uncertainty and confusion about mask-wearing, and described educating participants about the importance of mask-wearing.

Table 2 New COVID-19-related practices and changes to existing SSP delivery models/services

\begin{tabular}{|c|c|}
\hline & Practices/changes described by respondents \\
\hline New COVID-19-related practices & $\begin{array}{l}\text { Physical markers and reminders to encourage social distancing } \\
\text { Limiting the number of participants allowed in a space at one time } \\
\text { "No-contact" exchange/service delivery } \\
\text { Use of PPE (e.g., masks) and sanitization } \\
\text { Screening participants for COVID-19 symptoms } \\
\text { Providing COVID-19-related information and/or linkage to testing }\end{array}$ \\
\hline Changes to physical space or model & $\begin{array}{l}\text { Moving fixed-site models all or partially outdoors } \\
\text { Switching to or increasing mobile/delivery models }\end{array}$ \\
\hline Changes to supplies distributed & $\begin{array}{l}\text { Increasing number of syringes distributed per participant to reduce in-person contact } \\
\text { Increasing amount of other supplies distributed (e.g., naloxone, fentanyl testing strips) } \\
\text { Moving from one-for-one exchange to needs-based model } \\
\text { Encouraging more secondary distribution } \\
\text { Pre-packaging supplies to speed up interactions }\end{array}$ \\
\hline Changes to operational capacity & $\begin{array}{l}\text { Decrease in staff due to safety concerns, resource cuts, or reassignment due to COVID-19 } \\
\text { Decrease in volunteers, students and/or interns due to safety concerns and/or personal reasons } \\
\text { Decrease in hours of operation due to limited staff } \\
\text { Increase in hours of operation through expanded mobile/delivery services }\end{array}$ \\
\hline Changes to HIV and HCV testing & $\begin{array}{l}\text { Stopping HIV and HCV testing due to safety concerns and/or loss of testing supplies } \\
\text { Switching to self-administered/at-home HIV testing }\end{array}$ \\
\hline $\begin{array}{l}\text { Changes to substance use disorder treatment } \\
\text { linkage }\end{array}$ & $\begin{array}{l}\text { Treatment program staff no longer present at SSP } \\
\text { Shortening time spent discussing treatment with interested participants } \\
\text { Navigating new regulatory policies around medication treatment for opioid use disorder }\end{array}$ \\
\hline Changes to other services & $\begin{array}{l}\text { Stopping other services due to safety concerns (e.g., wound care, health education classes) } \\
\text { Adding other services (e.g., providing food/essentials, mailing address for stimulus check) } \\
\text { Stopping street outreach; some SSPs increased outreach through mobile/delivery efforts }\end{array}$ \\
\hline
\end{tabular}


"The uptake of face covering wearing among the general public is not super high here, so it's kind of like fighting against a head wind to get folks to understand, yeah, there are a lot of people who are making bad decisions for their health right now and we don't want you to be one of those people." (Respondent 28, program coordinator/manager, urban, West)

The availability of PPE and sanitization supplies varied; some respondents reported having adequate supplies and others reported having difficulty finding supplies. Some SSPs received PPE through their affiliation with health departments but noted health departments were prioritized below other entities (e.g., hospitals) for PPE receipt. SSPs also received supply donations and/or used masks made by staff/volunteers. While no respondents reported that lack of supplies resulted in stoppage of service provision, SSP staff sometimes reused PPE, used makeshift PPE (e.g., tying a bandana around their face) or went without sanitization supplies when none were available.

\section{Screening for COVID-19 varied across SSPs, and respondents described multiple barriers to screening}

About one-third of respondents reported that formal COVID19 screening was currently being conducted at their SSPs, and several reported having discontinued screening. Screening practices included asking about symptom checklists and/ or checking temperature. Screening was sometimes conducted inconsistently, e.g., only for participants requesting referral to clinical services. Some programs informally asked participants if they had been feeling sick. Though most respondents did not describe a protocol for positive screens, a few said these participants would be offered/referred to COVID-19 testing and/or have supplies brought to them outside; one reported they would do "no-contact" mobile delivery.

Respondents described several barriers to implementing screening. Taking time to screen was challenging in the context of trying to serve participants quickly to reduce COVID19 transmission risk. Some felt screening was not effective because respiratory symptoms are common in this population due to prevalence of smoking and chronic illness, because symptoms may take time to appear after infection, and/or because some COVID-19 cases are asymptomatic. Some felt a positive screen would not change service provision and was therefore not worth doing.

"We were [screening] in the beginning and then we just sort of stopped because...it was just like we're trying to make it really fast, and that's already kind of a known entity, right? So, our folks are mostly gonna come [to the SSP] anyway." (Respondent 3, program coordinator/ manager, urban, South)

A few respondents saw screening as conflicting with their approach to service delivery, expressing concern about treating participants "like they're damaged in any way" (Respondent 17, high-level administrator, urban, South) or "invad[ing] on their self-determination" (Respondent 24, program coordinator/manager, urban, South). One described how screening felt incompatible with their role as a peer of SSP participants.

"We don't really have any like, 'we're gonna take your temperature, we're gonna screen you,' like that. I'm really a peer...I have a lot of experience, I'm a former injection drug user, but it really gets into some weird areas where somebody with my lack of credentials starts taking people's temperature and it gets a little clinical." (Respondent 4, program coordinator/manager, rural, Northeast)

\section{Some SSPs provided additional COVID-19-related information or linkage to testing}

Some SSPs provided COVID-19-related information to participants, including strategies to avoid transmission and safer drug use during the pandemic. Respondents pointed out that SSPs are a trusted source of health information among their participants. Some SSPs provided information about testing locations, and a few offered tests in partnership with a health department or clinic.

\section{Changes were made to existing delivery models and services to accommodate COVID-19-related precautions}

Implementation of COVID-19-related practices was accompanied by several changes to SSPs' existing services, including changes to the physical space or delivery model, supplies distributed, operational capacity, HIV/HCV testing, SUD treatment linkage, and other services (Table 2). Of note, several respondents reported reductions in staff/volunteers due to individuals' concerns about transmission, resource cuts, and reassignment of staff due to COVID-19. In some cases, this resulted in reduced hours of operation.

\section{Concerns about the pandemic's impact on SSP services and the wellbeing of PWUD}

Respondents discussed their concerns related to the pandemic's impact on SSP services and the broader wellbeing of PWUD (Fig. 1). 


\section{Fewer PWUD may be accessing supplies to prevent transmission of drug use-related infections and overdose death}

Respondents had mixed perceptions of whether their SSP was reaching fewer, more, or the same number of participants compared to before the pandemic. However, some were concerned about the impact of reduced hours, decreased outreach efforts, and having lost contact with some participants while closed. A few had heard reports of increased syringe sharing. Further, some noted the difficulty of measuring how reach had been impacted, and some perceived their numbers had increased due to other SSPs closing making it challenging to understand impact on overall community reach. Some expressed concerns about having adequate funding to continue reliably providing services in the future.

"We are hearing about massive amounts of syringe sharing...from participants, they're saying it feels like the '90s again. So, we're expecting spikes in HIV and spikes in hepatitis $C$, and at the same time worrying and preparing for budget cuts for those programs...So, we might really be going back in time, which is horrific." (Respondent 8 , high-level administrator, urban, Northeast)

\section{Suspended HIV and HCV testing at SSPs may lead to decreased diagnosis}

Nearly all SSPs that had been providing HIV/HCV testing had stopped due to the pandemic. Reasons for this included concern about COVID-19 transmission, lack of time and staff to conduct testing, and sudden unavailability of testing supplies. Some SSPs had received explicit mandates to stop testing from outside entities (e.g., health departments). Many SSPs hoped to restore testing, a few were still providing testing with increased distancing and PPE precautions, and a few had implemented or were planning to implement self-administered or take-home testing. Some expressed concern that lack of HIV/HCV testing would lead to decreased diagnosis among PWUD.

"I think the biggest thing is, so, we haven't been able to access testing and provide testing...our numbers have consistently been like 1/3 of the people we test are hep $C$ positive. So that is an entire population of people we're missing now." (Respondent 26, program coordinator/manager, urban, Midwest)

\section{COVID-19 has created barriers to linking interested SSP participants to SUD treatment}

Many SSPs were still asking participants about interest in SUD treatment and/or linking them to treatment through referrals or integrated services. However, some respondents discussed how distancing precautions have reduced time available to discuss treatment with participants.

"Case managers do follow up with phone calls...but it's hard to promote services when you really don't get the chance to sit down with clients and talk for however long they need to talk about resources they need, about medication-assisted treatment." (Respondent 1, nurse, rural, West)
Fig. 1 Respondents' concerns and perspectives on opportunities to improve future services for PWUD

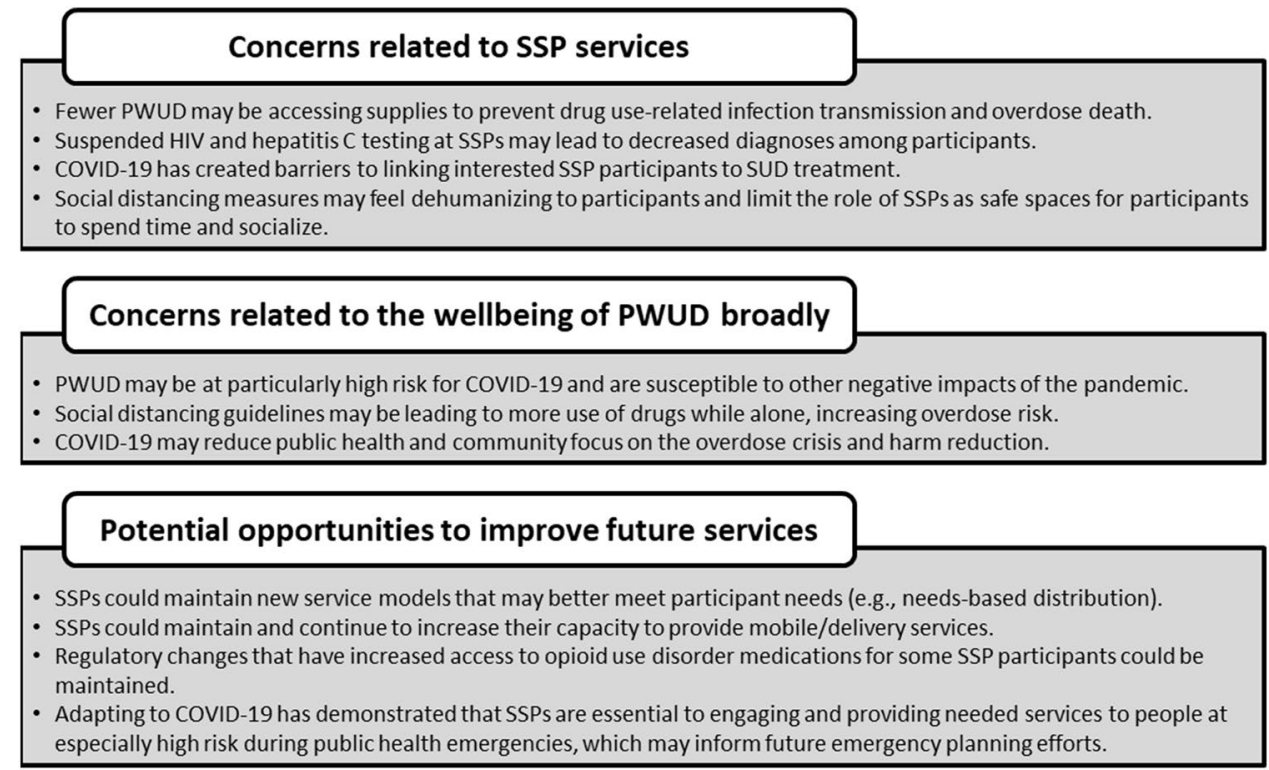


Additionally, some SSPs that previously had staff from treatment programs on site to facilitate linkage no longer had that resource, and some perceived that many treatment programs were not open or not taking new patients due to COVID-19.

\section{Social distancing measures may feel dehumanizing and limit the role of SSPs as safe spaces for participants}

Respondents expressed concern that social distancing hindered their ability to emotionally support participants and may feel dehumanizing to participants. Several noted that SSPs often play an important role in participants' lives as a safe space to spend time and socialize, and that distancing protocols were limiting this source of support. Some said SSP staff were reaching out to participants by telephone when possible.

\begin{abstract}
"We're all about making people feel like they matter and that they're human because the world is telling them the opposite, so social distancing was very hard for us to implement...we turned all of our distribution sites from being spaces where people could enter to places where people could only access supplies through windows." (Respondent 8, high-level administrator, urban, Northeast)
\end{abstract}

\section{PWUD may be at high risk for COVID-19 and other negative impacts of the pandemic}

Respondents were concerned that many PWUD have limited ability to protect themselves from COVID-19 exposure (particularly those living in congregate settings), have multiple underlying health conditions putting them at risk for more severe COVID-19 disease progression, and face multiple barriers to healthcare access including stigmatization and lack of insurance. Some worried that many participants did not understand the severity of COVID-19.

"I think my biggest concern is that we're already working with people who are physically pretty vulnerable... there's a lot of skepticism about COVID. People, that comes up every session, somebody will say, 'You know, is this thing for real?"' (Respondent 2, nurse, urban, West)

Respondents were also concerned about other pandemic impacts on PWUD, including the closure of public spaces and services, increases in unemployment and homelessness, and the impact of isolation on mental health.
Social distancing guidelines may lead to more use of drugs while alone, increasing overdose risk

Respondents noted that guidelines promoting social distancing may lead to more solitary drug use, which increases overdose risk. Some perceived that overdoses had increased during the pandemic based on reports from participants and/or available data.

"One thing that we always talk about with folks is not using alone. And we knew that that was just going to be a reality for a lot of folks. So, trying to push out a lot of education on how folks can stay as safe as possible." (Respondent 16, high-level administrator, urban, Northeast)

\section{COVID-19 may reduce public health and community focus on the overdose crisis and harm reduction}

Some respondents were concerned about the pandemic decreasing focus on responding to the overdose crisis and harm reduction services, and that funding would be redirected from these efforts.

"My other worry is that...we've been operating within the opioid crisis and the overdose crisis, and we were starting to get a little bit of traction around those issues, that everything's gonna be pretty much wiped away and the progress that we've had towards...supervised injection and supervised consumption, which is what we really need, is gonna be put on the back burner." (Respondent 8 , high-level administrator, urban, Northeast)

\section{Potential opportunities to improve SSP and other services for PWUD}

Respondents also discussed potential opportunities to improve future services for PWUD resulting from changes made in response to COVID-19 (Fig. 1).

\section{Some programs have shifted to syringe distribution practices that may better meet participant needs}

While many SSPs already had needs-based syringe distribution models, some changed from one-for-one to needs-based models in response to COVID-19. Some respondents said they would eventually return to one-for-one models, and some were unsure what would happen after the pandemic. Several were concerned they would not have adequate funding and supplies to keep up current distribution rates. Nevertheless, some noted this was an unexpected opportunity to enact their preferred model and better meet participant needs in contexts where needs-based models had not been 
supported. Respondents also noted an increase in secondary distribution as a positive impact.

"I know it's fairly conservative in our area, so I feel like we're just really lucky that we're able to make [a needs-based model] happen...this gives us a chance to reestablish with [participants] that we are part of their community and we're here for them." (Respondent 2, nurse, urban, West)

\section{Some SSPs have increased their capacity to provide mobile/delivery services, which may increase access}

Several SSPs initiated or expanded mobile/delivery services in response to COVID-19. Respondents felt that increasing capacity to provide these services was a positive impact, and some noted this had allowed them to reach new participants. Some hoped these services would be sustained post-pandemic, and one mentioned that state policies requiring SSPs to be designated as either fixed-site or mobile may be changed to allow more flexibility, noting the possibility that "the state will just find that this has been a very useful and very needed way to get syringes to people that need them." (Respondent 21, program coordinator/manager, rural, Northeast).

\section{Changes to MOUD regulations have improved access}

Respondents described how increased flexibility in MOUD regulations has improved access for SSP participants, mentioning decreased barriers to buprenorphine (e.g., telephone initiations, not requiring urine drug screens), and increased take-home doses of methadone. Respondents hoped these changes would remain after the pandemic. However, some perceived that not all treatment programs were implementing new guidelines, e.g., some were "still making people come in more frequently and give urine samples" (Respondent 3 program coordinator/manager, urban, South).

"The barrier is much, much lower for our participants to get into [MOUD] programs than it used to be, which used to be you had to physically go to a place and jump through all these hoops, basically, that made it more difficult for our participants to enter those sorts of things." (Respondent 28, program coordinator/manager, urban, West)

\section{Adapting to COVID-19 demonstrated the essential role SSPs play in engaging and providing needed services to PWUD during public health emergencies}

Respondents felt that by rapidly adapting and continuing to provide services during the pandemic, SSPs demonstrated their essential nature and commitment to meeting the needs of PWUD. Several perceived that continuing services strengthened the trust PWUD have in their programs, and that participants viewed them as trusted sources of COVID19-related information. A few hoped that moving forward, SSPs will be recognized as key partners in preparing for and responding to public health emergencies in order to engage community members who may be at especially high risk.

"SSPs really should be, you know, some of the first partners around the table to discuss response, because they have such a privileged relationship and rapport built with homeless folks, folks experiencing unstable housing, people who are participating in sex work... who are going to be more affected by outbreaks or crises like this." (Respondent 7, state-level leadership, urban, South)

\section{Discussion}

SSPs rapidly adapted services to continue operating during the COVID-19 pandemic in the context of varied state/local regulations and processes to obtain essential service designation. COVID-19-related precautions have been accompanied by several changes to SSPs' existing services such as increasing mobile/delivery models, increasing supplies distributed per participant, and decreasing additional services including suspending $\mathrm{HIV} / \mathrm{HCV}$ testing. While respondents noted opportunities to improve current and future services, they had concerns regarding negative impacts of the pandemic on SSP services and the overall wellbeing of PWUD. Many of these findings, including rapid implementation of changes, decreased staff/volunteers, and adoption of evidence-based distribution strategies, align with another recent qualitative study of SSPs [21]. Recommendations for health departments, policymakers, funders, community leaders, and other key stakeholders to support SSPs during and after the COVID-19 pandemic are summarized in Fig. 2.

These findings build on our previous assessment [19], and highlight needed support for SSPs to prevent the spread of COVID-19 as they deliver essential services during the pandemic. A consistent supply of PPE and sanitization supplies is needed to protect staff and participants during service delivery, and to allow SSPs to better distribute these supplies to PWUD, until vaccination rates are sufficiently high among PWUD. SSPs are trusted sources of COVID-19 information among many PWUD and could be important venues for COVID-19 vaccine distribution in this population. SSPs should be involved in vaccine distribution planning to determine the feasibility and acceptability of vaccine distribution to participants and to address potential concerns.

SSPs should also be involved in future emergency planning given their engagement with a population that may be 
particularly at-risk during pandemics and other emergencies. As SSPs provide essential, life-saving services for PWUD, they should be rapidly and clearly designated as essential public health infrastructure during emergencies to avoid closures. This may require improved communication with nonprofit/community-based SSPs without formal health department affiliations. These findings highlighted the importance of SSPs having internal decision-making processes to facilitate rapid response and the ability to adjust; however, making clear jurisdiction-level guidance for emergencies available to SSPs may aid this process. This guidance can be informed by lessons SSPs are learning during the COVID-19 pandemic. The range of SSPs' responses to COVID-19 documented here may inform future research and interventions to guide best practices for SSP emergency preparedness and response. For example, our team's ongoing work to support SSPs through evaluation and technical assistance has been directly informed by these findings.

Some SSPs may transform current adaptations into improved future services. Those that have switched from one-for-one to needs-based distribution models and/or increased secondary distribution-recommended best practices to increase adequate syringe coverage [27, 28]may demonstrate to community leaders, funders or other decision-makers that these practices should be permanently implemented, and efforts to maintain evidence-based practices should be supported by these stakeholders. However, some respondents felt these models would not be sustainable given their current resources, so increased financial support will likely be needed. Additionally, some programs are building their capacity to provide mobile/delivery services which may increase access for populations at increased risk [29].

Respondents discussed several concerns with serious implications for the health of PWUD, including expected increases in blood-borne pathogen transmission and overdose. To combat these multiple health crises, it is essential that SSPs be supported in their efforts to restore services to pre-COVID levels, and to expand their services to meet the needs of PWUD. This will require adequate funding to support increased supply distribution per participant and could also include support for outreach strategies to reach PWUD not currently connected to SSP services. Additionally, it is necessary to restore $\mathrm{HIV} / \mathrm{HCV}$ testing services in these populations, which may involve expanding self-administered/take-home testing and/or supporting SSPs in implementing safety protocols for in-person testing. Respondents also raised concerns that SSPs' role as a safe space for PWUD to spend time and receive social/emotional support had been substantially reduced. Innovative strategies could be developed for SSPs to maintain connection with participants during the pandemic, such as setting up safe outdoor gatherings for participants and staff. Finally, in the wake of a worsening overdose crisis $[4,6-8,30]$, SSPs must be supported in increasing naloxone distribution, and in continued provision of/linkage to opioid agonist medications, which reduce overdose risk for people with OUD [31, 32]. This should include maintaining regulatory changes that have increased access to buprenorphine and methadone among SSP participants. Expansion of services and an expanded role for SSPs (e.g., involvement in vaccine distribution and future emergency response planning) will likely require increasing the SSP staff/volunteer workforce, which several respondents reported has decreased due to individual's concerns about transmission and resource cuts. Therefore, both increased financial investment in SSPs and prioritization of staff/volunteer safety are needed.

This study has limitations. The sample may not be generalizable to all U.S. SSPs. Notably, although SSPs that had closed and not reopened at the time of recruitment were eligible to be included in the sample, they may have
Fig. 2 Recommendations to support SSPs during and after the COVID-19 pandemic

\section{Support SSPs in safely restoring and strengthening services}

Encourage and maintain evidence-based practices including needs-based distribution, secondary exchange, and mobile and/or delivery models.

- Identify and prioritize strategies for safely restoring HIV and HCV testing.

- Identify and prioritize strategies for ensuring initial and sustained linkages to substance use disorder treatment, including through maintaining regulatory changes that have increased access to MOUD and expanded telehealth.

- Identify and support innovative strategies for SSPs to maintain connection with and support participants, particularly unhoused and isolated participants.

- Ensure SSPs have adequate PPE supplies for staff and participants.

- Provide sufficient funding to SSPs to safely adapt and maintain services.

- Engage SSPs in COVID-19 vaccine distribution.

\section{Prepare for future potential public health emergencies}

- Automatically and clearly designate SSPS as essential services.

Engage SSPs in strategic planning for response efforts.

- Include SSPS in distribution of emergency supplies to PWUD.

- Ensure sufficient funding for SSPS to effectively respond to future emergencies.

Ensure SSPs are incorporated into local public health infrastructure. 
been less likely to respond. Efforts to support SSPs that have not reopened, and to understand their barriers to reopening, are needed. These data were collected relatively early in the pandemic, and therefore the context, available resources, and strategies employed by SSPs may have changed following data collection. Additionally, responses may have been impacted by the specific date of the interview within the study period (e.g., April vs. June) and the modality that was available to the interviewee (videoconferencing software vs. telephone). Overall, these interviews provide insight into a pivotal moment for SSPs as they first adapted services in response the pandemic, and assessment is underway to understand ongoing service provision.

Future research should examine differences across region, urban/rural location, and organizational characteristics in COVID-19-related changes to SSP services and associated impacts. Research should also seek to further understand the impact of community support or lack thereof on SSPs' operations and ability to respond to emergencies, as well as the personal experiences of SSP staff/volunteers in order to better support this workforce. Finally, this study was limited to perspectives of SSP leadership/staff. A recent qualitative study found that PWUD in rural Oregon made several suggestions for SSPs that align with those reported here, including needs-based distribution, increasing mobile/delivery services, and increasing secondary exchange [33]. Future studies should continue to examine the perspectives of PWUD, which are essential to improving services and adequately meeting the needs of PWUD during and after the pandemic.

\section{Conclusions}

Qualitative interviews with 31 U.S. SSP staff and leadership found that most SSPs rapidly adapted their services to continue operations during the COVID-19 pandemic. COVID-19-related precautions included social distancing, use of PPE and sanitization supplies, and in some programs, COVID-19 screening, and these precautions were accompanied by several changes to existing services. Respondents discussed some areas of opportunity, including the possibility of maintaining evidence-based best practices (e.g., needs-based distribution and mobile/delivery models) after the pandemic. However, they also raised important concerns including reduced reach of SSPs, suspended HIV/ HCV testing, barriers to SUD treatment linkage, and the pandemic's impact on PWUD more broadly. These findings can inform efforts to better support SSPs in restoring and expanding services while protecting staff and participants from COVID-19, as well as the potential role of SSPs in engaging PWUD during the COVID-19 response and future emergencies, efforts that will require financially supporting SSPs and prioritizing safety of the SSP workforce. The pandemic may be indirectly increasing transmission of drug use-related infections and overdose death, thereby posing serious, immediate threats to PWUD beyond COVID-19 itself. Safe restoration and expansion of SSP services and continued efforts to combat the overdose crisis must remain top public health priorities.

Supplementary Information The online version contains supplementary material available at https://doi.org/10.1007/s10461-021-03332-7.

Acknowledgements The authors thank the SSP staff and leadership who participated in interviews. Thank you to Emmanuel Rodriguez for providing the Spanish translation of the abstract.

Author Contributions The study was conceived and designed by Sara Glick, Don Des Jarlais, Stephanie Prohaska and Paul LaKosky. Sampling and recruitment were facilitated by Stephanie Prohaska and Paul LaKosky. Data were collected by Sara Glick, Maria Corcorran, Alexa Juarez, and Noah Frank. Analyses were led by Emily Williams, Elizabeth Austin and Sara Glick, and conducted by Madeline Frost, Elsa Sweek, and Maria Corcorran. Madeline Frost led preparation of the manuscript; all authors contributed revisions to the manuscript and read and approved the final manuscript.

Funding This project was conducted with support from the Centers for Disease Control and Prevention (Grant No. 5 NU65 PS92368501). Ms. Frost is supported by a predoctoral training award from the Veterans Affairs Puget Sound Research and Development Service. Dr. Corcorran was supported by a training grant from the National Institute of Diabetes and Digestive and Kidney Diseases during this project (Grant No. 5 T32 DK007742-22). The opinions expressed in this work are the authors' and do not necessarily reflect those of the institutions, funders, the Centers for Disease Control and Prevention, the Department of Veterans Affairs, the National Institutes of Health, or the United States Government.

\section{Declarations}

Conflict of interest The authors have no relevant financial or non-financial interests to disclose.

Ethical Approval The University of Washington Institutional Review Board determined that this study was not human subjects research.

Consent to Participate All participants provided verbal consent to participate in the interview.

\section{References}

1. Wakeman SE, Green TC, Rich J. An overdose surge will compound the COVID-19 pandemic if urgent action is not taken. Nat Med. 2020;26(6):819-20.

2. Volkow ND. Collision of the COVID-19 and Addiction Epidemics. Ann Intern Med. 2020;173(1):61-3.

3. Chayama KL, Ng C, McNeil R. Calls for access to safe injecting supplies as a critical public health measure during the COVID-19 pandemic. J Addict Med. 2020;14(5):e142-3. 
4. Centers for Disease Control and Prevention Health Alert Network. Increase in Fatal Drug Overdoses Across the United States Driven by Synthetic Opioids Before and During the COVID-19 Pandemic. Accessed January 12, 2020 from: https://emergency. cdc.gov/han/2020/han00438.asp?ACSTrackingID=USCDC_511DM44961\&ACSTrackingLabel=HAN\%20438\%20-\%20General\% 20Public\&deliveryName=USCDC_511-DM44961. 2020.

5. Ahmad FB RL, Sutton P. Provisional drug overdose death counts. National Center for Health Statistics. Accessed March 17, 2021 at: https://www.cdc.gov/nchs/nvss/vsrr/drug-overdose-data.htm. 2021.

6. Slavova S, Rock P, Bush HM, Quesinberry D, Walsh SL. Signal of increased opioid overdose during COVID-19 from emergency medical services data. Drug Alcohol Depend. 2020;214:108176.

7. Ochalek TA, Cumpston KL, Wills BK, Gal TS, Moeller FG. Nonfatal opioid overdoses at an urban emergency department during the COVID-19 pandemic. JAMA. 2020;324(16):1673-4.

8. American Medical Association Advocacy Resource Center. Issue brief: Reports of increases in opioid- and other drug-related overdose and other concerns during COVID pandemic. Accessed October 20, 2020 from: https://www.ama-assn.org/system/files/ 2020-10/issue-brief-increases-in-opioid-related-overdose.pdf. 2020.

9. Des Jarlais DC, Marmor M, Paone D, Titus S, Shi Q, Perlis T, et al. HIV incidence among injecting drug users in New York City syringe-exchange programmes. Lancet. 1996;348(9033):987-91.

10. Palmateer N, Kimber J, Hickman M, Hutchinson S, Rhodes T, Goldberg D. Evidence for the effectiveness of sterile injecting equipment provision in preventing hepatitis $\mathrm{C}$ and human immunodeficiency virus transmission among injecting drug users: a review of reviews. Addiction. 2010;105(5):844-59.

11. Behrends CN, Nugent AV, Des Jarlais DC, Frimpong JA, Perlman DC, Schackman BR. Availability of HIV and HCV on-site testing and treatment at syringe service programs in the United States. J Acquir Immune Defic Syndr. 2018;79(2):e76-8.

12. Peiper NC, Clarke SD, Vincent LB, Ciccarone D, Kral AH, Zibbell JE. Fentanyl test strips as an opioid overdose prevention strategy: Findings from a syringe services program in the Southeastern United States. Int J Drug Policy. 2019;63:122-8.

13. Lambdin BH, Bluthenthal RN, Wenger LD, Wheeler E, Garner B, Lakosky P, et al. Overdose education and naloxone distribution within syringe service programs-United States, 2019. MMWR Morb Mortal Wkly Rep. 2020;69(33):1117-21.

14. Leonard L, DeRubeis E, Pelude L, Medd E, Birkett N, Seto J. "I inject less as I have easier access to pipes": injecting, and sharing of crack-smoking materials, decline as safer crack-smoking resources are distributed. Int J Drug Policy. 2008;19(3):255-64.

15. Hood JE, Banta-Green CJ, Duchin JS, Breuner J, Dell W, Finegood B, et al. Engaging an unstably housed population with low-barrier buprenorphine treatment at a syringe services program: Lessons learned from Seattle. Washington Subst Abus. 2020;41(3):356-64.

16. Bachhuber MA, Thompson C, Prybylowski A, Benitez JM, Mazzella SM, Barclay D. Description and outcomes of a buprenorphine maintenance treatment program integrated within Prevention Point Philadelphia, an urban syringe exchange program. Subst Abus. 2018;39(2):167-72.

17. Tookes H, Bartholomew TS, St Onge JE, Ford H. The university of miami infectious disease elimination act syringe services program: A blueprint for student advocacy, education, and innovation. Acad Med. 2021;96(2):213-7.

18. Bluthenthal RN. Syringe exchange as a social movement: a case study of harm reduction in Oakland. California Subst Use Misuse. 1998;33(5):1147-71.
19. Glick SN, Prohaska SM, LaKosky PA, Juarez AM, Corcorran MA, Des Jarlais DC. The impact of COVID-19 on syringe services programs in the United States. AIDS Behav. 2020;24(9):2466-8.

20. Bartholomew TS, Nakamura N, Metsch LR, Tookes HE. Syringe services program (SSP) operational changes during the COVID19 global outbreak. The International journal on drug policy. 2020. https://doi.org/10.1016/j.drugpo.2020.102821.

21. Wenger LD, Kral AH, Bluthenthal RN, Morris T, Ongais L, Lambdin BH. Ingenuity and Resiliency of Syringe Service Programs on the Front Lines of the Opioid Overdose and COVID-19 Crises. Transl Res. 2021. https://doi.org/10.1016/j.trsl.2021.03. 011.

22. Wang QQ, Kaelber DC, Xu R, Volkow ND. COVID-19 risk and outcomes in patients with substance use disorders: analyses from electronic health records in the United States. Mol Psychiatry. 2020;26(1):30-9.

23. Beebe J. Rapid Assessment Process: An Introduction. Walnut Creek, CA: Altmira Press; 2001.

24. Hamilton A. Rapid Turn-Around: Tips for Speeding Up Qualitative Projects. 5th Annual Planning for Qualitative Research: Design, Analysis and Software Intergration; University of North Carolina Chapel Hill, NC. 2014.

25. McMullen CK, Ash JS, Sittig DF, Bunce A, Guappone K, Dykstra $\mathrm{R}$, et al. Rapid assessment of clinical information systems in the healthcare setting: an efficient method for time-pressed evaluation. Methods Inf Med. 2011;50(4):299-307.

26. Taylor B, Henshall C, Kenyon S, Litchfield I, Greenfield S. Can rapid approaches to qualitative analysis deliver timely, valid findings to clinical leaders? A mixed methods study comparing rapid and thematic analysis. BMJ Open. 2018;8(10):e019993.

27. Bluthenthal RN, Ridgeway G, Schell T, Anderson R, Flynn $\mathrm{NM}$, Kral AH. Examination of the association between syringe exchange program (SEP) dispensation policy and SEP clientlevel syringe coverage among injection drug users. Addiction. 2007;102(4):638-46.

28. New York City Department of Health and Mental Hygiene. Recommended best practices for effective syringe exchange programs in the United States: Results of a consensus meeting. Accessed 24 Oct 2020 from: http://www.santacruzhealth.com/Portals/7/Pdfs/ SEP\%20Recs\%20-\%20Consensus\%20Meeting.pdf. 2009.

29. Miller CL, Tyndall M, Spittal P, Li K, Palepu A, Schechter MT. Risk-taking behaviors among injecting drug users who obtain syringes from pharmacies, fixed sites, and mobile van needle exchanges. J Urban Health. 2002;79(2):257-65.

30. Mann B. Sharp Rise In Drug Overdose Deaths Seen During 1st Few Months Of Pandemic. NPR. Accessed October 20, 2020 from: https://www.npr.org/sections/coronavirus-live-updates/ 2020/10/14/923721544/sharp-rise-in-drug-overdose-deaths-seenduring-1st-few-months-of-pandemic. 2020.

31. Pierce M, Bird SM, Hickman M, Marsden J, Dunn G, Jones A, et al. Impact of treatment for opioid dependence on fatal drugrelated poisoning: a national cohort study in England. Addiction. 2016;111(2):298-308.

32. Wakeman SE, Larochelle MR, Ameli O, Chaisson CE, McPheeters JT, Crown WH, et al. Comparative effectiveness of different treatment pathways for opioid use disorder. JAMA Netw Open. 2020;3(2):e1920622.

33. Seaman A, Leichtling G, Stack E, Gray M, Pope J, Larsen JE, et al. Harm reduction and adaptations among PWUD in rural oregon during COVID-19. AIDS Behav. 2021;25(5):1331-9.

Publisher's Note Springer Nature remains neutral with regard to jurisdictional claims in published maps and institutional affiliations. 